\title{
Photo-induced excitonic spin dynamics in GaAs
}

\author{
M. I. Miah ${ }^{1,2}$ and Lubna Naheed ${ }^{3}$ \\ ${ }^{1}$ Department of Physics, University of Chittagong, Chittagong-4331, Bangladesh. \\ ${ }^{2}$ Queensland Micro- and Nanotechnology Centre, Griffith University, Nathan, \\ Brisbane, QLD 4111, Australia. \\ ${ }^{3}$ Applied Physics Department, Pakistan Council of Scientific and Industrial Research, \\ Labs Complex, Lahore, Pakistan. \\ Corresponding Author Email: m.miah@griffith.edu.au
}

\begin{abstract}
The dynamics of the optical orientated excitonic spins in semiconductor quantum wells has been investigated using opposite circular polarizations of the photoluminescence (PL) caused by the spin-dependent phase-space filling. The PL excitonic spin polarization estimated from the two different polarization conditions is found to strongly depend on the strength of the electric bias as well as on the size of the wells. With an increase of the electric bias field, the excitonic PL spin polarization increases, giving a maximum value of $\sim 90 \%$ at the fields higher than $\sim 2.5 \mathrm{~V}$. However, the PL spin polarization decreases with increasing the size of the well as well as increasing crystal temperature. The results as well as possible reasons responsible for the effects are discussed.
\end{abstract}

Keywords: Exciton; Quantum well; Spin kinetics 


\section{Introduction}

Spin is closely related to magnetic phenomena, and as an intrinsic magnetic moment is associated with the spin, electron spin provides an extra degree of freedom, socalled the spin degree of freedom, for the electron to interact with a magnetic field [1].

The idea of utilizing the electron spin in semiconductor devices leads to the growth of the field "spintronics", where both charge and spin degrees of freedoms play an important role in realizing operations and functionalities [2,3]. The basic study dedicated to spintronics is to understand the interaction between the particle spin and its solid-state environments in order to make useful devices based on the acquired knowledge [4]. This may result in the development of novel electronic devices with advantages of e.g., nonvolatility (data retainer), reduced power consumption (energy saver), smaller size (larger integration density) and faster operation (higher speed).

One of the basic requirements necessary in developing semiconductor spintronic devices is the efficient generation (injection) of spins in (into) semiconductor heterostructures such that they can be transported reliably (without loss of the spin polarization) over reasonable distances, i.e. distances that are comparable to the device dimensions [5-9]. In order to understand this, semiconductor-based heterostructures have been studied. For example, optically detected magnetic resonance in zero field and in a finite magnetic field was used to study the excitons in type-II GaAs/AlAs quantum wells, where the spectra were analysed using the spin Hamiltonian for the quasi-two-dimensional indirect excitons [5]. Subsequent investigations for photoexcited carrier dynamics and excitonic energy in quantum dots 
and for optical orientation of electron spins under electric bias in semiconductor quantum wells (QWs) are also present [10-12].

Here in the present investigation, we focus on the study of the dynamics of the optical orientated excitonic spins under drift in QWs by circularly polarized photoluminescence (PL) measurements. In QWs, spins are generated by exciting their heavy-hole valence bands by a left/right circularly polarized light [13]. The left and right circularly polarized light excited PL in dependences of the applied external bias, thickness $t$ of the QWs and crystal temperature are measured. Their dependences are explored in the present systematic study of, in particular, well thickness and temperature.

\section{Samples and experimental details}

Experimental samples were GaAs quantum wells, grown on the Si-doped GaAs substrate using the MBE method. They were $\mathrm{Al}_{0.3} \mathrm{Ga}_{1-0.3} \mathrm{As}$ QWs with different well thicknesses. The barrier width was $\sim 20 \mathrm{~nm}$. A negative external bias with different field strengths (up to $-4 \mathrm{~V}$ ) was applied. For the application of the external bias, the top surface of the sample was coated with a semitransparent electrode. We measured the photoluminescence excited by ps right circularly polarized light pulses of a tunable Ti:sapphire laser with a repetition rate of $76 \mathrm{MHz}$ using a streak camera. The PL spectra of the QW structures displayed intense excitonic lines with a half-width of 2-3 meV. Approximately of the same value was the Stokes shift between the exciton peaks in the absorption and PL spectra. The circularly polarized PL was excited directly to the exciton absorption band under normal incidence and was detected with the small long-wavelength shift $(\sim 2 \mathrm{meV})$ to minimize the polarization losses. The 
circularly polarized PL was detected in the backward direction. Measurements were performed at different temperatures by placing the samples in a liquid helium temperature-regulated cryostat. The PL polarization was calculated by defining it as the ratio of the difference of the signals of the right and left circularly polarized PL to their sum. A schema of the experimental setup is shown in Fig. 1.

\section{Results and discussion}

The excitonic PL in dependences of the external electric bias and quantum well thickness was measured for the left and right polarization conditions. There was a difference between the different polarization conditions, which is caused by spindependent phase-space filling $[14,15]$. The resulting PL polarization, as measured with pulses with the same and opposite circular polarization, as a function of the time delay was calculated.

From our pump-probe delay, the exitonic PL polarization or the exciton spin was found to decay in a sub-nanosecond (Fig. 2). The dependence of the PL polarization on the external electric bias is shown in Fig. 3. As can be seen, with an increase of the applied electric bias, the excitonic PL spin polarization increases. However, it gets saturated to $\sim 90 \%$ at the fields higher than $\sim 2.5 \mathrm{~V}$.

In the presence of the external electric bias, the electric field reduces the electron-hole exchange coupling by spatially separating the charges, and as a result, the interaction of the hole spin with phonons becomes stronger than the exchange interaction. This leads to breakage of the coupling between the electron and hole spins. As a consequence, the hole spin exhibits fast relaxation, while the electron spin holds its 
orientation, providing the slow component in the polarization decay. Relaxation can result in the PL depolarization due to a flip of the exciton spin as a whole or independent flips of the electron and hole spins $[17,18]$. In the first case, the decay time of the PL polarization is directly determined by the exciton spin relaxation rate. If the carrier spins relax independently, the fast flip of the hole spin does not affect the PL polarization. In this case, the decay of the PL polarization is controlled by the relaxation of the long-lived electron spin. The above analysis reveals that the biasinduced changes in the PL kinetics are related to the transition from dynamics of the exciton spin to that of the independent electron and hole spins. The application of the bias to the QWs weakens the exchange interaction between the electron and hole spins [19]. This is because the recombination in QWs is reduced upon the application of a bias due to a decreased wave function overlap of the electrons and holes. In the absence of the bias, the exchange coupling exceeds the spin-phonon interaction originated from photo-induced electron-phonon anharmonicity [20], and the main relaxation mechanism is given by the exciton spin flips.

Dependence of PL polarization on the thickness of QW was studied. The results are shown in Fig. 4. As can be seen, the PL polarization decreases as the thickness increases. The breakage of the spin coupling strongly depends on the well thickness, where with increasing thickness of the well, the exchange interaction increases, and consequently, the hole spin relaxation rare decreases. In addition, in thin QWs, the efficiency of spatial separation of the electron and hole by the electric field is smaller than in thick wells. Owing to this joint action, a relatively small change in the well thickness increases the external bias required for breakage of the coupling of the electron and hole spins [21]. This fact explains the noticeable changes in the PL 
polarization and its kinetics, as observed in the experiment (Fig. 3). The same effect was observed by others [22-24].

The excitonic PL polarization at different crystal temperatures is shown in Fig. 5. As can be seen, the PL polarization decreases with increasing sample temperature. The observed temperature dependence might be due to the temperature-dependent bandgap shift of the SQW materials. The temperature dependence of the direct bandgap, determined from the absorption edge, of a zinc-blende semiconductor, such as GaAs, can be described by $E_{g}=E_{g}^{0}-m T^{2} /(n+T)$, where $E_{g}^{0}$ is the bandgap at $0 \mathrm{~K}$, and $m$ and $n$ are temperature independent constant parameters [25]. As can be seen, the band-gap energy decreases as the sample temperature increases. Thus the circularly polarized light with photon energy slightly lower than the light-hole-band to conduction band energy gap might be able to excite some of the carriers from the light-hole valence band due to the band-narrowing effect at higher temperatures, and consequently the excitonic spin polarization would decrease.

\section{Conclusions}

Optically induced excitonic spins dynamics in quantum wells was investigated using opposite circular polarizations of the PL. The PL excitonic spin polarization estimated from the two different polarization conditions was found to strongly depend on the strength of the electric bias as well as on the size of the wells. With an increase of the electric bias field, the excitonic PL spin polarization was found to increase. However, it decreased with increasing the size of the well. This was mainly because of the breakage of the spin coupling as it strongly depended on the well thickness, with increasing the thickness of the well, the exchange interaction increased. It was also 
found that the excitonic spin polarization decreased with increasing the crystal temperature. This might be due to the temperature-dependent energy bandgap effect on the optical spin generation.

\section{References}

[1] Spin Electronics, M. Ziese and M. J. Thornton, Eds., Vol. 569 (SpringerVerlag, Heidelberg, 2001).

[2] M. I. Dyakonov and A. V. Khaetskii, Spin Hall Effect (Spin Physics in Semiconductors, M. I. Dyakonov, ed., Springer-Verlag, Berlin, 2008).

[3] Semiconductor Spintronics and Quantum Computation, D.D. Awschalom, D. Loss, and N. Samarth, Eds. (Springer, Berlin, 2002).

[4] M. I. Miah, J. Optoelectron. Adv. Mater. 10, 2487 (2008).

[5] H. W. van Kesteren, E. C. Cosman, W. A. J. A. van der Poel, and C. T. Foxon, Phys. Rev. B 41, 5283 (1990).

[6] J. M. Kikkawa, and D.D. Awschalom, Phys. Rev. Lett. 80, 4313 (1998).

[7] S. A. Wolf, D.D. Awschalom, R.A. Buhrman, J.M. Daughton, S. von Molnár, M.L. Roukes, A.Y. Chtchelkanova, and D.M. Treger, Science 294, 1488 (2001).

[8] R. I. Dzhioev, V. L. Korenev, B. P. Zakharchenya, D. Gammon, A. S. Bracker, J. G. Tischler, and D. S. Katzer, Phys. Rev. B 66, 153409 (2002).

[9] X. Lou, C. Adelmann, C. A. Crooker, E. S. Garlid, J. Zhang, K. S. M. Reddy, S. D. Flexner, C. J. Palmstrøm, and P. A. Crowell, Nat. Phys. 3, 197 (2007).

[10] S. P. Dash, S. Sharma, R. S. Patel, M. P. de Jong, and R. Jansen, Nature 462, 491 (2009).

[11] E. Ilczuk, K. P. Korona, A. Babiński, and J. Kuhl, Acta Phys. Pol. A 100, 379 (2001). 
[12] S. Raymond, S. Studenikin, A. Sachrajda, Z. Wasilewski, S. J. Cheng, W. Sheng, P. Hawrylak, Adam Babiński, M. Potemski, G. Ortner, and M. Bayer, Phys. Rev. Lett. 92, 187402 (2004).

[13] G. E. Pikus, and A. N. Titkov, in Optical Orientation, Modern Problems in Condensed Matter Science (F. Meier and B. P. Zakharchenya, Eds.), Vol. 8 (North-Holland, Amsterdam, 1984).

[14] G. Lampel and C. Weisbuch, Solid State Comm. 16, 877 (1975).

[15] I. A. Avrutsky and A. V. Vosmishev, Phys. Low-Dim. Struct. 10/11, 257 (1995).

[16] T. Wang, A. Li, and Z. Tan, Proc. SPIE, Vol. 6838, 683814 (2007).

[17] M. I. Miah, Appl. Phys. Lett. 94, 182106 (2009).

[18] S. R. Nabi, Exchange, Bull. Mater. Sci. 23, 34 (2000).

[19] G. E. Pikus and A. N. Titkov, In Optical Orientation (Modern Problems in Condensed Matter Science, Vol. 8, North-Holland, Amsterdam, 1984).

[20] I. V. Kityk, J. Non-Crystalline Solids 292, 184 (2001).

[21] M. I. Miah, J. Phys. D: Appl. Phys. 41, 185503 (2008).

[22] A. P. Heberle, W. W. Ruhle, and K. Ploog, Phys. Lett. 72, 3887 (1994).

[23] S. Hallstein, J. D. Berger, M. Hilpert, H. C. Schneider, W.W. Ruhle, F. Jahnke, S. W. Koch, H. M. Gibbs, G. Khitrova, and M. Oestreich, Phys. Rev. B 56, R7076 (1997).

[24] L. C. O. Dacal and J. A. Brum, Phys. Rev. B 65, 115324 (2002).

[25] Y. Varshni, Physica 34, 149 (1967). 


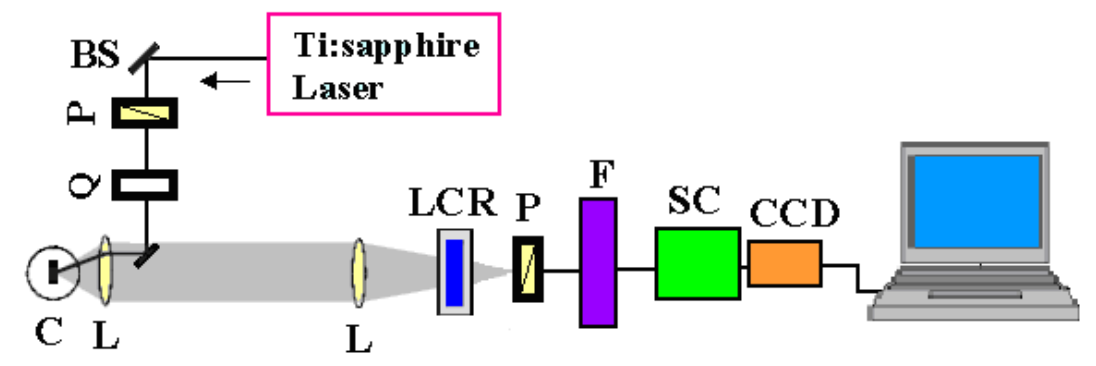

Figure 1. A schema of the experimental setup for the investigation of the excitonic spin dynamics. 


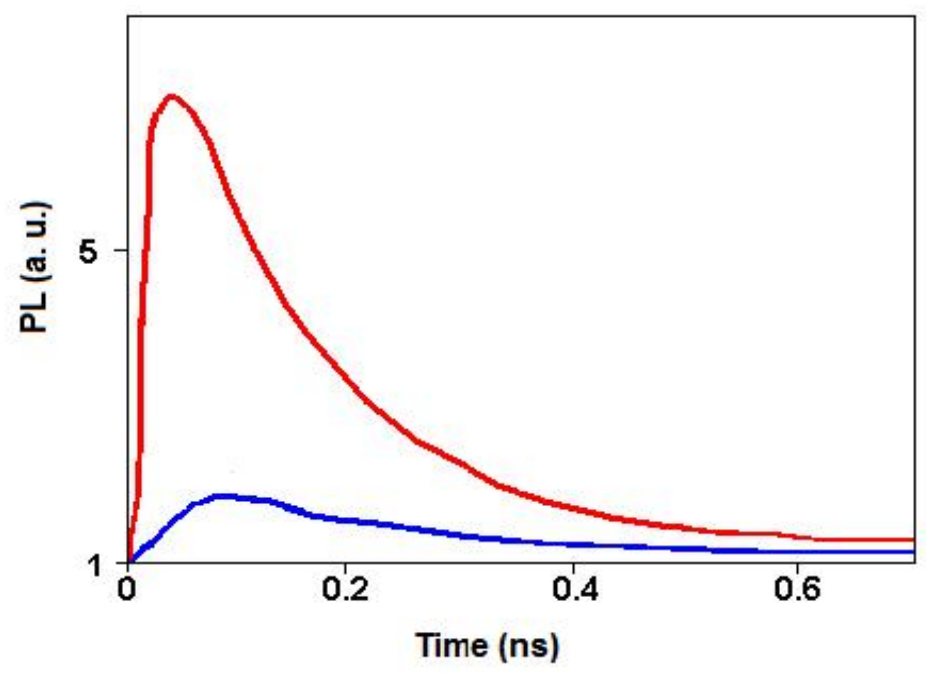

Figure 2. Typical emission intensities with the same (top) and opposite (bottom) circularly polarized light excitations. 


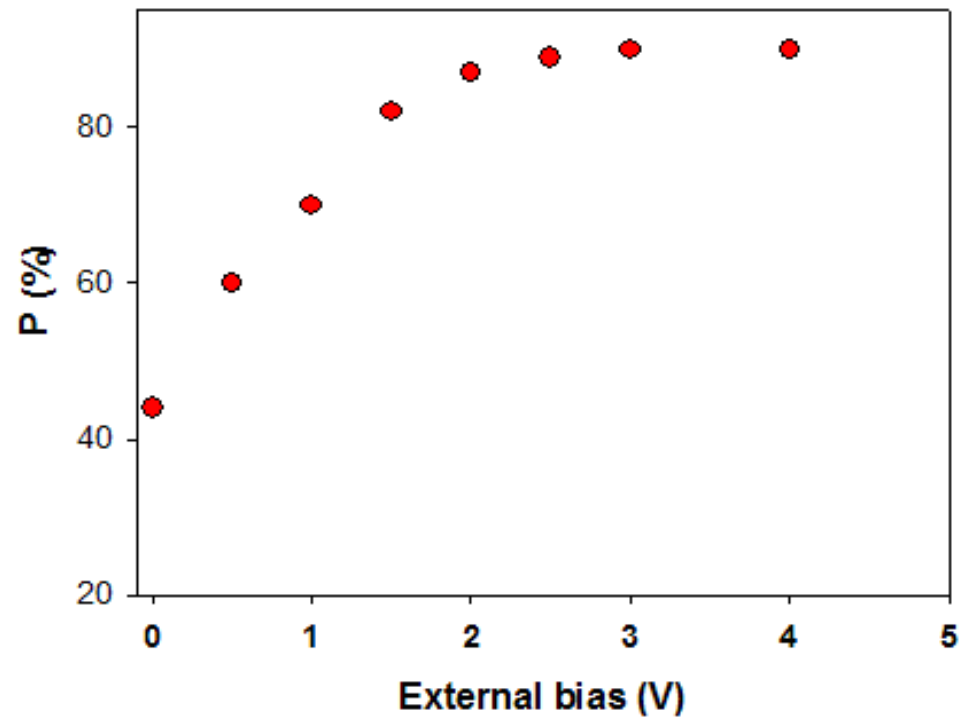

Figure 3. Excitonic spin polarization as a function of the applied electric bias at liquid helium temperature. 


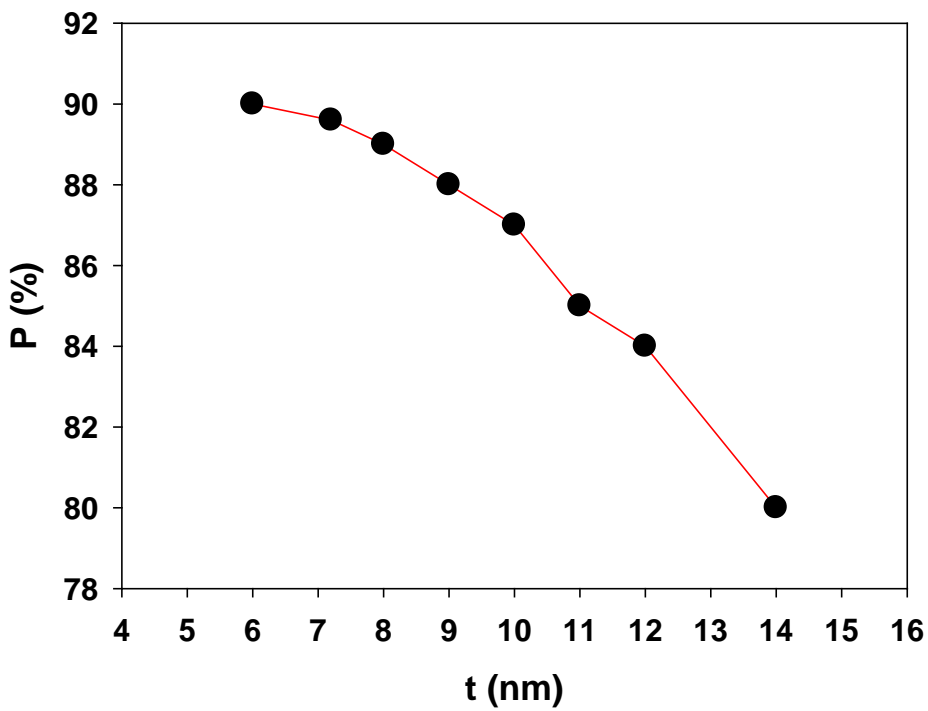

Figure 4. Excitonic spin polarization as a function of the quantum well thickness at liquid helium temperature. 


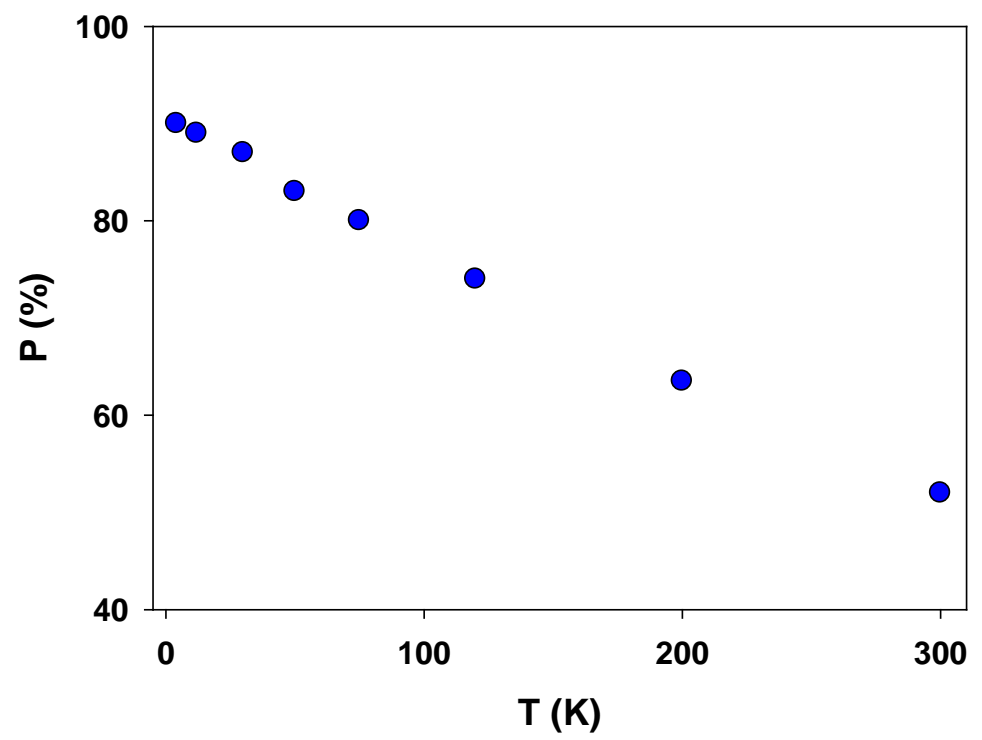

Figure 5. Excitonic spin polarization as a function of the crystal temperature for a sample with $\mathrm{t}=6 \mathrm{~nm}$. 